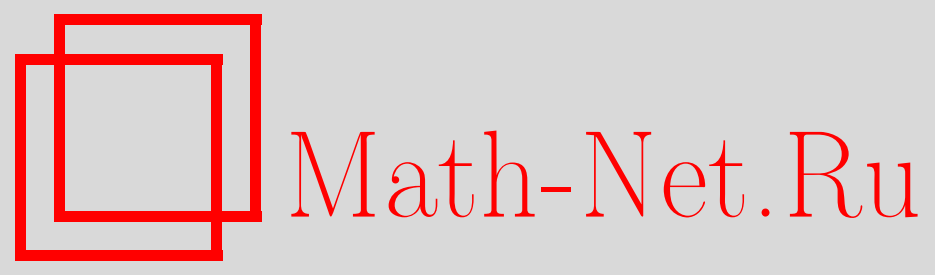

А. П. Янковский, Асимптотический анализ решения нелинейной задачи нестационарной теплопроводности слоистых анизотропных неоднородных оболочек при граничных условиях первого рода на лицевых поверхностях, Вестн. Сам. гос. техн. ун-та. Сер. Физ.-мат. науки, 2014, выпуск 1(), 168-185

DOI: https://doi.org/10.14498/vsgtu1281

Использование Общероссийского математического портала MathNet.Ru подразумевает, что вы прочитали и согласны с пользовательским соглашением

http://www . mathnet.ru/rus/agreement

Параметры загрузки:

IP: 3.93 .64 .190

26 апреля 2023 г., 12:36:23

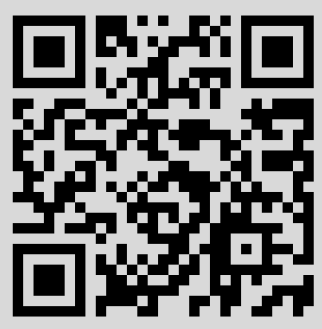


УДК 536.21

\title{
АСИМПТОТИЧЕСКИЙ АНАЛИЗ РЕШЕНИЯ НЕЛИНЕЙНОЙ ЗАДАЧИ НЕСТАЦИОНАРНОЙ ТЕПЛОПРОВОДНОСТИ СЛОИСТЫХ АНИЗОТРОПНЫХ НЕОДНОРОДНЫХ ОБОЛОЧЕК ПРИ ГРАНИЧНЫХ УСЛОВИЯХ ПЕРВОГО РОДА НА ЛИЦЕВЫХ ПОВЕРХНОСТЯХ
}

\section{А. П. Янковский}

Институт теоретической и прикладной механики им. С. А. Христиановича СО РАН, Россия, 630090, Новосибирск, ул. Институтская, 4/1.

\begin{abstract}
Сбормулирована задача теплопроводности для слоистьх оболочек, состоящих из термочувствительных анизотропных неоднородных слоёв, при граничных условиях общего вида. Термочувствительность материалов слоёв описъвается линейной зависимостью их теплофизических характеристик от температурь. Проведено обезразмеривание уравнения теплопроводности, граничных условий и условий теплового сопряжения на границах контакта между слоями. Выделены два малых параметра в безразмерных соотношениях: теплобизический, характеризующий степень термочувствительности материалов слоёв, и геометрический, характеризующий относительную толщину оболочки. Проведена последовательная рекурсия безразмерных соотношений сначала по теплофизическому малому параметру, а затем по геометрическому. Первый тип рекурсии позволил линеаризовать задачу теплопроводности, а на основе второго типа рекурсии построено внешнее асимптотическое разложение решения задачи нестационарной теплопроводности слоистых анизотропных неоднородных оболочек при граничных условиях первого рода на лищевых поверхностях. Проанализированы получающиеся двумерные разрешающие уравнения и исследованы асимптотические свойства решений задачи теплопроводности.
\end{abstract}

Ключевые слова: теплопроводность, термочувствительность, асимптотический анализ, слоистье оболочки, анизотропия, неоднородностъ.

Введение. Тонкостенные пластины и оболочки с пространственными структурами армирования - типичный конструктивный элемент в инженерной практике [1-6 и др.], исследование которого приводит к необходимости учёта в расчётах неоднородности композиционного материала и анизотропии самого общего вида. Функциональное назначение конструкции зачастую требует использование слоистой структуры изделия [7,8 и др.] с анизотропными и неоднородными материалами слоёв вследствие различного типа 3Dармирования $[9,10]$ и других технологических факторов.

Так как современные тонкостенные конструкции (особенно аэрокосмического назначения) подвергаются интенсивному термосиловому воздействию,

ISSN: 2310-7081 (online), 1991-8615 (print); doi: http://dx.doi.org/10.14498/vsgtu1281 (C) 2014 Самарский государственный технический университет.

Образец цитирования: А. П. Я н к в с к и й, "Асимптотический анализ решения нелинейной задачи нестационарной теплопроводности слоистых анизотропных неоднородных оболочек при граничных условиях первого рода на лицевых поверхностях" // Becmн. Сам. гос. техн. ун-та. Сер. Физ.-мат. науки, 2014. № 1(34). С. 168-185. doi: 10.14498/vsgtu1281.

Сведения об авторе: Андрей Петрович Янковский (д.ф.-м.н., проф.), ведущий научный сотрудник, лаб. физики быстропротекающих процессов.

E-mail address: lab4nemir@rambler.ru 
актуальной становится задача изучения температурных полей в них. Для уточнения распределения температурных полей в изделиях, подвергнутых интенсивному тепловому воздействию, необходимо учитывать зависимость теплофизических характеристик их материалов от температуры, что приводит к необходимости изучения нелинейных задач теплопроводности.

В связи с этим настоящее исследование посвящено построению внешнего асимптотического разложения основного температурного поля нелинейной задачи нестационарной теплопроводности слоистых анизотропных неоднородных оболочек и пластин при граничных условиях I рода на лицевых поверхностях с учётом термочувствительности материалов слоёв, в отличие от [11], где рассмотрен случай, когда на лицевых поверхностях заданы граничные условия III рода (при средних и больших числах Био) или смешанные граничные условия (I и II рода на разных лицевых поверхностях).

\section{1. Постановка нелинейной задачи теплопроводности слоистых анизотроп-} ных оболочек. Рассматривается тонкая оболочка, состоящая из $M$ анизотропных неоднородных слоёв, возможно, переменной толщины. Свяжем с оболочкой криволинейную ортогональную систему координат $\bar{x}_{1}, \bar{x}_{2}, \bar{x}_{3}$ так, чтобы отсчётная поверхность $\bar{x}_{3}=0$ совпадала с одной из лицевых поверхностей оболочки (например, внутренней), а поверхности $\bar{x}_{3}=\bar{H}_{m}=$ const $>0$ определяли границы контакта между $m$-тым и $(m+1)$-м слоями $(m=1,2, \ldots, M)$; значение $\bar{x}_{3}=\bar{H}_{0} \equiv 0$ задаёт отсчётную поверхность, $\bar{x}_{3}=\bar{H}_{M} \equiv \bar{H}=$ $=$ const $>0$ - другую лицевую поверхность; слои последовательно пронумерованы от отсчётной поверхности к противоположной лицевой поверхности. Параметры Ламе $\bar{A}_{1}, \bar{A}_{2}$ непрерывны всюду в оболочке и имеют гладкость, которая потребуется в процессе рассуждений; параметр Ламе $\bar{A}_{3}$ на границах контакта слоёв может испытывать разрыв первого рода (т.е. $\bar{A}_{3}=\bar{A}_{3}^{(m)}$ при $\left.\bar{H}_{m-1}<\bar{x}_{3}<\bar{H}_{m}, 1 \leqslant m \leqslant M\right)$, внутри каждого $m$-того слоя этот параметр имеет гладкость, которая потребуется в процессе рассуждений. (В случае слоёв постоянной толщины координата $\bar{x}_{3} \geqslant 0$ задаёт расстояние от произвольной точки оболочки до отсчётной поверхности, при этом $\bar{A}_{3}=1$.) На границах между слоями выполняются условия идеального (полного) теплового контакта.

При сделанных предположениях уравнение нестационарной теплопроводности $m$-того слоя имеет вид [7]

$$
\begin{aligned}
& \bar{c}^{(m)}\left(\bar{T}^{(m)}\right) \bar{\rho}^{(m)} \frac{\partial \bar{T}^{(m)}}{\partial \bar{t}}= \\
&=\frac{1}{\bar{A}_{1} \bar{A}_{2} \bar{A}_{3}} \sum_{i=1}^{3} \frac{\partial}{\partial \bar{x}_{i}}\left(\sum_{j=1}^{3} \frac{\bar{A}_{1} \bar{A}_{2} \bar{A}_{3} \bar{\lambda}_{i j}^{(m)}\left(\bar{T}^{(m)}\right)}{\bar{A}_{i} \bar{A}_{j}} \frac{\partial \bar{T}^{(m)}}{\partial \bar{x}_{j}}\right)+ \\
& \quad+\bar{Q}^{(m)}\left(\bar{x}_{1} \bar{x}_{2} \bar{x}_{3} \bar{t}\right), \quad \bar{H}_{m-1} \leqslant \bar{x}_{3} \leqslant \bar{H}_{m}, \quad 1 \leqslant m \leqslant M,
\end{aligned}
$$

где $\bar{T}^{(m)}$ - отклонение температуры $m$-того слоя от температуры естественного состояния конструкции $\bar{T}_{*} ; \bar{Q}^{(m)}-$ плотность мощности внутренних источников тепла в $m$-том слое; $\bar{\lambda}_{i j}^{(m)}$ - коэффициенты теплопроводности материала $m$-того слоя; $\bar{c}^{(m)}$ - удельная теплоёмкость материала $m$-того слоя; $\bar{\rho}^{(m)}-$ объёмная плотность материала $m$-того слоя; $\bar{t}$ - время. Здесь и далее размер- 
ные функции и величины будем помечать сверху чертой, а соответствующие им безразмерные функции и величины - обозначать теми же символами, но без черты.

В случае учёта термочувствительности материалов слоёв коэффициенты $\bar{\lambda}_{i j}^{(m)}, \bar{c}^{(m)}$ в $(1)$ зависят от $\bar{T}^{(m)}$. В первом приближении эту зависимость можно принять линейной $[12,13]$ :

$$
\begin{aligned}
& \bar{\lambda}_{i j}^{(m)}\left(\bar{T}^{(m)}\right)=\bar{k}_{i j}^{(m)}+\bar{\beta}_{i j}^{(m)} \bar{T}^{(m)}, \quad i, j=\overline{1,3}, \\
& \bar{c}^{(m)}\left(\bar{T}^{(m)}\right)=\bar{c}_{*}^{(m)}+\bar{c}_{* *}^{(m)} \bar{T}^{(m)},
\end{aligned}
$$

где $\bar{k}_{i j}^{(m)}, \bar{c}_{*}^{(m)}$ - коэффициенты теплопроводности и удельная теплоемкость материала $m$-того слоя при температуре естественного состояния конструкции $\bar{T}_{*}\left(\bar{T}^{(m)}=0\right) ; \bar{\beta}_{i j}^{(m)}, \bar{c}_{* *}^{(m)}$ - коэффициенты температурной зависимости, характеризующие термочувствительность материала $m$-того слоя. В общем случае величины $\bar{k}_{i j}^{(m)}, \bar{\beta}_{i j}^{(m)}, \bar{c}_{*}^{(m)}, \bar{c}_{* *}^{(m)}, \bar{\rho}^{(m)}$ могут зависеть от пространственных переменных в силу неоднородности материала $m$-того слоя.

На поверхностях $\bar{x}_{3}=\bar{H}_{m}$ контакта $m$-того и $(m+1)$-го слоёв должны выполняться условия сопряжения решения по тепловому потоку и температуре $[7,12]$

$$
\begin{gathered}
\sum_{i=1}^{3} \frac{\bar{\lambda}_{3 i}^{(m)}}{\bar{A}_{i}} \frac{\partial \bar{T}^{(m)}}{\partial \bar{x}_{i}}=\sum_{i=1}^{3} \frac{\bar{\lambda}_{3 i}^{(n)}}{\bar{A}_{i}} \frac{\partial \bar{T}^{(n)}}{\partial \bar{x}_{i}}, \quad \bar{T}^{(m)}=\bar{T}^{(n)}, \\
\bar{x}_{3}=\bar{H}_{m}, \quad n=m+1, \quad 1 \leqslant m \leqslant M-1 ;
\end{gathered}
$$

на лицевых поверхностях оболочки $\left(\bar{x}_{3}=0, \bar{H}\right)$ заданы граничные условия общего вида $[7,12]$

$$
\begin{aligned}
& \left.\beta^{(-)} \sum_{i=1}^{3} \frac{\bar{\lambda}_{3 i}^{(1)}}{\bar{A}_{i}} \frac{\partial \bar{T}^{(1)}}{\partial \bar{x}_{i}}\right|_{\bar{x}_{3}=0}=\gamma^{(-)} \bar{Q}^{(-)}\left(\bar{x}_{1}, \bar{x}_{2}, \bar{t}\right)+ \\
& +\bar{\delta}^{(-)} \bar{\alpha}_{(-)}\left(\bar{T}^{(1)}\left(\bar{x}_{1}, \bar{x}_{2}, 0, \bar{t}\right)-\bar{T}_{\infty}^{(-)}\left(\bar{x}_{1}, \bar{x}_{2}, \bar{t}\right)\right), \\
& -\left.\beta^{(+)} \sum_{i=1}^{3} \frac{\bar{\lambda}_{3 i}^{(M)}}{\bar{A}_{i}} \frac{\partial \bar{T}^{(M)}}{\partial \bar{x}_{i}}\right|_{\bar{x}_{3}=\bar{H}}=\gamma^{(+)} \bar{Q}^{(+)}\left(\bar{x}_{1}, \bar{x}_{2}, \bar{t}\right)+ \\
& +\bar{\delta}^{(+)} \bar{\alpha}_{(+)}\left(\bar{T}^{(M)}\left(\bar{x}_{1}, \bar{x}_{2}, \bar{H}, \bar{t}\right)-\bar{T}_{\infty}^{(+)}\left(\bar{x}_{1}, \bar{x}_{2}, \bar{t}\right)\right),
\end{aligned}
$$

где $\bar{Q}^{( \pm)}$- заданные на лицевых поверхностях проекции вектора теплового потока на направление внешней нормали; $\bar{\alpha}_{( \pm)}-$коэффициенты конвективного теплообмена с окружающей средой на внешней $(+)$ и внутренней $(-)$, т. е. отсчётной, поверхностях оболочки; $\bar{T}_{\infty}^{( \pm)}$- температура окружающей среды со стороны внешней $(+)$ и внутренней $(-)$ лицевой поверхности оболочки; $\beta^{( \pm)}, \gamma^{( \pm)}, \bar{\delta}^{( \pm)}$- функции переключения, позволяющие задавать тот или иной тип граничных условий на внешней $(+)$ и внутренней $(-)$ лицевых поверхностях. 
На торцевой поверхности $\bar{S}$ (кромке) оболочки также заданы граничные условия, аналогичные (4):

$$
\begin{aligned}
& -\beta \sum_{i=1}^{3} \bar{n}_{i}\left(\sum_{j=1}^{3} \frac{\bar{\lambda}_{i j}^{(m)}}{\bar{A}_{j}} \frac{\partial \bar{T}^{(m)}}{\partial \bar{x}_{j}}\right)=\gamma \bar{q}_{n}^{(m)}(\bar{S}, \bar{t})+ \\
& +\delta \bar{\alpha}^{(m)}\left(\bar{T}^{(m)}(\bar{S}, \bar{t})-\bar{T}_{\infty}(\bar{S}, \bar{t})\right), \quad\left(\bar{x}_{1}, \bar{x}_{2}, \bar{x}_{3}\right) \in \bar{S}, \bar{t} \geqslant \bar{t}_{0}, 1 \leqslant m \leqslant M,
\end{aligned}
$$

где $\bar{n}_{i}$ - компоненты вектора единичной нормали к торцевой поверхности оболочки; $\bar{q}_{n}^{(m)}$ - заданный тепловой поток через торцевую поверхность $m$-того слоя; $\bar{\alpha}^{(m)}$ - коэффициент теплообмена по закону Ньютона между $m$-тым слоем и окружающей средой на торцевой поверхности; $\bar{T}_{\infty}-$ температура окружающей среды со стороны торцевой поверхности (или температура торцевой поверхности, смотря по смыслу); $\beta, \gamma, \delta$ - функции переключения, позволяющие задавать тот или иной тип граничных условий на торцевой поверхности.

В момент времени $\bar{t}_{0}$ в $m$-тым слое задано начальное условие

$$
\bar{T}^{(m)}\left(\bar{x}_{1}, \bar{x}_{2}, \bar{x}_{3}, \bar{t}_{0}\right)=\bar{T}_{000}^{(m)}\left(\bar{x}_{1}, \bar{x}_{2}, \bar{x}_{3}\right), \quad 1 \leqslant m \leqslant M
$$

где $\bar{T}_{000}^{(m)}$ - известная функция.

Обезразмерим соотношения (1)-(6). С этой целью введём безразмерные независимые переменные

$$
\begin{aligned}
& \bar{A}_{i} d \bar{x}_{i}=\bar{L} A_{i} d x_{i}, \quad i=1,2, \\
& \bar{A}_{3} d \bar{x}_{3}=\bar{H}_{*} A_{3} d x_{3}, \quad t=\bar{t} / \bar{t}_{*} \quad\left(\bar{t}_{*}>0, \quad \bar{L}=\min (\bar{R}, \bar{a})\right),
\end{aligned}
$$

где $\bar{R}$ - характерный радиус кривизны отсчётной поверхности оболочки; $\bar{a}-$ характерный размер оболочки в плане (для пологих оболочек и искривленных панелей); $\bar{H}_{*}$ - характерная толщина оболочки; $\bar{t}_{*}-$ характерное время, в течение которого рассматривается процесс нестационарной теплопроводности.

Уравнение (1) обезразмерим умножением на $A_{1} A_{2} A_{3} \bar{H}_{*}^{2} /\left(\bar{\lambda}_{*} \bar{T}_{*}\right)$, тогда с учётом (7) получим

$$
\begin{aligned}
& \varepsilon^{2} C^{(m)} \partial_{t} T^{(m)}=\varepsilon^{2} \sum_{i=1}^{2} \sum_{j=1}^{2} \partial_{i}\left(\lambda_{i j}^{(m)} \partial_{j} T^{(m)}\right)+\varepsilon \sum_{i=1}^{2} \partial_{i}\left(\lambda_{i 3}^{(m)} \partial_{3} T^{(m)}\right)+ \\
& \quad+\partial_{3}\left[\varepsilon \sum_{i=1}^{2} \lambda_{3 i}^{(m)} \partial_{i} T^{(m)}+\lambda_{33}^{(m)} \partial_{3} T^{(m)}\right]+\varepsilon^{2} Q^{(m)}(\mathbf{x}, t), \quad 1 \leqslant m \leqslant M,
\end{aligned}
$$

где согласно (2)

$$
\begin{aligned}
& \lambda_{i j}^{(m)}=k_{i j}^{(m)}+\eta \beta_{i j}^{(m)} T^{(m)}, \quad i, j=\overline{1,3}, \\
& C^{(m)}=C_{*}^{(m)}+\eta C_{* *}^{(m)} T^{(m)}, \quad \mathbf{x}=\left\{x_{1}, x_{2}, x_{3}\right\}
\end{aligned}
$$

$\varepsilon=\bar{H}_{*} / \bar{L}$ - малый геометрический параметр; $\eta$-малый теплофизический параметр [12], характеризующий степень термочувствительности материалов 
слоёв (при $\eta \rightarrow 0$ получаем случай линейной теплопроводности слоистой оболочки при отсутствии термочувствительности материалов слоёв); $\partial_{i}$ - оператор частного дифференцирования по безразмерной пространственной переменной $x_{i}(i=\overline{1,3}) ; \partial_{t}$ - оператор частного дифференцирования по безразмерному времени $t$.

Умножением на $A_{1} A_{2} \bar{H}_{*} /\left(\bar{\lambda}_{*} \bar{T}_{*}\right)$ обезразмерим первое условие сопряжения (3), а второе условие $(3)$ - делением на $\bar{T}_{*}=$ const:

$$
\begin{aligned}
& \varepsilon\left(\lambda_{31}^{(m)} \partial_{1} T^{(m)}+\lambda_{32}^{(m)} \partial_{2} T^{(m)}\right)+\lambda_{33}^{(m)} \partial_{3} T^{(m)}= \\
& =\varepsilon\left(\lambda_{31}^{(n)} \partial_{1} T^{(n)}+\lambda_{32}^{(n)} \partial_{2} T^{(n)}\right)+\lambda_{33}^{(n)} \partial_{3} T^{(n)}, \quad T^{(m)}=T^{(n)}, \\
& x_{3}=H_{m}, n=m+1,1 \leqslant m \leqslant M-1 ;
\end{aligned}
$$

граничные условия (4) обезразмерим умножением на $A_{1} A_{2} \bar{H}_{*} /\left(\bar{\lambda}_{*} \bar{T}_{*}\right)$, а $(5)-$ умножением на $A_{1} A_{2} A_{3} \bar{H}_{*} /\left(\bar{\lambda}_{*} \bar{T}_{*}\right)$ :

$$
\begin{gathered}
\beta^{(-)}\left[\varepsilon\left(\lambda_{31}^{(1)} \partial_{1} T^{(1)}+\lambda_{32}^{(1)} \partial_{2} T^{(1)}\right)+\lambda_{33}^{(1)} \partial_{3} T^{(1)}\right]= \\
=\varepsilon \gamma^{(-)} Q^{(-)}+\varepsilon \delta^{(-)} \alpha_{(-)}\left(T^{(1)}-T_{\infty}^{(-)}\right), \quad x_{3}=0 ; \\
-\beta^{(+)}\left[\varepsilon\left(\lambda_{31}^{(M)} \partial_{1} T^{(M)}+\lambda_{32}^{(M)} \partial_{2} T^{(M)}\right)+\lambda_{33}^{(M)} \partial_{3} T^{(M)}\right]= \\
=\varepsilon \gamma^{(+)} Q^{(+)}+\varepsilon \delta^{(+)} \alpha_{(+)}\left(T^{(M)}-T_{\infty}^{(+)}\right), \quad x_{3}=H ; \\
-\beta \varepsilon \sum_{i=1}^{3} \sum_{j=1}^{2} n_{i} \lambda_{i j}^{(m)} \partial_{j} T^{(m)}-\beta \sum_{i=1}^{3} n_{i} \lambda_{i 3}^{(m)} \partial_{3} T^{(m)}=\varepsilon \gamma q_{n}^{(m)}+ \\
+\varepsilon \delta \alpha^{(m)}\left(T^{(m)}-T_{\infty}\right), \quad \mathbf{x} \in S, t \geqslant t_{0}, 1 \leqslant m \leqslant M ;
\end{gathered}
$$

начальное условие (6) обезразмерим делением на $\bar{T}_{*}=$ const:

$$
T^{(m)}\left(\mathbf{x}, t_{0}\right)=T_{000}^{(m)}(\mathbf{x}), \quad 1 \leqslant m \leqslant M .
$$

В соотношениях (8)-(14) с учётом (2) использованы следующие формулы обезразмеривания и обозначения:

$$
\begin{aligned}
& T^{(m)}=\bar{T}^{(m)} / \bar{T}_{*}, \quad \lambda_{i j}^{(m)}=A_{1} A_{2} A_{3} \bar{\lambda}_{i j}^{(m)} /\left(A_{i} A_{j} \bar{\lambda}_{*}\right), \\
& k_{i j}^{(m)}=A_{1} A_{2} A_{3} \bar{k}_{i j}^{(m)} /\left(A_{i} A_{j} \bar{\lambda}_{*}\right), \quad \eta_{*} \beta_{i j}^{(m)}=A_{1} A_{2} A_{3} \bar{T}_{*} \bar{\beta}_{i j}^{(m)} /\left(A_{i} A_{j} \bar{\lambda}_{*}\right), \\
& Q^{(m)}=A_{1} A_{2} A_{3} \bar{L}^{2} \bar{Q}^{(m)} /\left(\bar{\lambda}_{*} \bar{T}_{*}\right), \quad T_{\infty}^{( \pm)}=\bar{T}_{\infty}^{( \pm)} / \bar{T}_{*}, \\
& Q^{( \pm)}=A_{1} A_{2} \bar{L} \bar{Q}^{( \pm)} /\left(\bar{\lambda}_{*} \bar{T}_{*}\right), \quad \alpha_{( \pm)}=\bar{L} \bar{\alpha}_{( \pm)} / \bar{\lambda}_{*}, \\
& T_{\infty}=\bar{T}_{\infty} / \bar{T}_{*}, \quad T_{000}^{(m)}=\bar{T}_{000}^{(m)} / \bar{T}_{*}, \quad q_{n}^{(m)}=A_{1} A_{2} A_{3} \bar{L}_{n}^{(m)} /\left(\bar{\lambda}_{*} \bar{T}_{*}\right), \\
& \alpha^{(m)}=A_{1} A_{2} A_{3} \bar{L}^{(m)} / \bar{\lambda}_{*}, \quad C^{(m)}=A_{1} A_{2} A_{3} \bar{L}^{2} \bar{c}^{(m)} \bar{\rho}^{(m)} /\left(\bar{\lambda}_{*} \bar{t}_{*}\right), \\
& C_{*}^{(m)}=A_{1} A_{2} A_{3} \bar{L}^{2} \bar{c}_{*}^{(m)} \bar{\rho}^{(m)} /\left(\bar{\lambda}_{*} \bar{t}_{*}\right), \\
& \eta_{*} C_{* *}^{(m)}=A_{1} A_{2} A_{3} \bar{L}^{2} \bar{T}_{*} \bar{c}_{* *}^{(m)} \bar{\rho}^{(m)} /\left(\bar{\lambda}_{*} \bar{t}_{*}\right), \\
& t_{0}=\bar{t}_{0} / \bar{t}_{*}, \quad \delta^{( \pm)}=A_{1} A_{2} \bar{\delta}^{( \pm)}, \quad n_{i}=A_{i} \bar{n}_{i}, \quad i, j=1,2,3,1 \leqslant m \leqslant M ;
\end{aligned}
$$


$\bar{\lambda}_{*}$ - характерное значение коэффициента теплопроводности материалов слоёв оболочки (например, максимальная по слоям величина наибольшего из главных значений тензора коэффициентов теплопроводности $\bar{\lambda}_{i j}^{(m)}$ при температуре естественного состояния $\left.\bar{T}_{*}\right) ; \eta_{*}$ - конкретное значение малого параметра $\eta$, при котором решается рассматриваемая задача теплопроводности оболочки (значение $\eta_{*}$ выбирается, например, так, чтобы наибольшие значения величин $k_{i j}^{(m)}, \eta_{*} \beta_{i j}^{(m)}$ и $C_{*}^{(m)}, \eta_{*} C_{* *}^{(m)}$ были одного порядка или сопоставимы); $C^{(m)}$ - безразмерная теплоемкость (характерное значение времени $\bar{t}_{*}$ в (8), (15) выбрано так, чтобы значения величин $C^{(m)}, C_{*}^{(m)}, \eta_{*} C_{* *}^{(m)}$ были порядка единицы).

Если считать, что изменению малого геометрического параметра $\varepsilon$ соответствует изменение толщины оболочки $\bar{H}_{*}$ (толщины слоёв при этом изменяются пропорционально изменению $\bar{H}_{*}$ ) при фиксированной геометрии отсчётной поверхности конструкции (при фиксированном характерном размере $\bar{L}$ ), то основные функции и величины, приведенные в (15), имеют следующие асимптотические свойства:

$$
\begin{aligned}
& k_{i j}^{(m)}=O(1), \quad \beta_{i j}^{(m)}=O(1), \quad i, j=\overline{1,3} ; \\
& Q^{(m)}=O(1), \quad T_{\infty}^{( \pm)}=O(1), \quad Q^{( \pm)}=O(1), \quad T_{\infty}=O(1), \\
& q_{n}^{(m)}=O(1), \quad \alpha^{(m)}=O(1), \quad C_{*}^{(m)}=O(1), \quad C_{* *}^{(m)}=O(1), \\
& T_{000}^{(m)}=O(1) \quad \text { при } \quad \varepsilon \rightarrow 0 \quad\left(\text { аналогично при } \quad \eta \rightarrow 0 \quad \text { и } \quad \alpha_{( \pm)} \rightarrow 0\right) .
\end{aligned}
$$

Соотношения (8)-(14) формально совпадают с безразмерными уравнениями теплопроводности для слоистых анизотропных неоднородных пластин, поэтому дальнейшие рассуждения справедливы как для оболочек, так и для пластин.

Согласно $[14,15]$, в зависимости от условий конвективного теплообмена в граничных условиях общего вида (11), (12) безразмерные коэффициенты $\alpha_{( \pm)}$, характеризующие критерий Био [16] на лицевых поверхностях, могут иметь значения порядка единицы, а могут быть большими и малыми величинами по сравнению с единицей. Следовательно, при определенных условиях теплообмена на лицевых поверхностях безразмерные числа Био $\alpha_{( \pm)}$(см. (15)) можно рассматривать как независимые от $\varepsilon$ и $\eta$ малые или большие параметры.

Таким образом, следует раздельно рассматривать случаи больших и малых значений чисел Био на лицевых поверхностях оболочки.

\section{2. Асимптотический анализ нелинейной задачи теплопроводности слои-} стых оболочек и пластин. В настоящем исследовании рассмотрим лишь случай задания на лицевых поверхностях граничных условий I рода, т.е. при бесконечно больших числах Био $\left(\alpha_{( \pm)} \rightarrow \infty\right.$, см. $\left.(11),(12)\right)$.

Так как согласно (9) при $\eta \rightarrow 0$ не происходит вырождения дифференциальных операторов в соотношениях начально-краевой задачи (8)-(14), для её линеаризации можно использовать следующее асимптотическое разложение:

$$
T^{(m)}(\mathbf{x}, t) \sim \sum_{k=0}^{\infty} T_{k}^{(m)}(\mathbf{x}, t) \eta^{k}, \quad 1 \leqslant m \leqslant M, \quad t \geqslant t_{0} .
$$


Подставим (17) в (8)-(14) и соберем слагаемые при одинаковых степенях $\eta$, тогда получим следующую цепочку равенств для определения функций $T_{k}^{(m)}(\mathbf{x}, t)$ :

$$
\begin{aligned}
& \varepsilon^{2} C_{*}^{(m)} \partial_{t} T_{k}^{(m)}+\varepsilon^{2} C_{k}^{(m)}= \\
&=\varepsilon^{2} \sum_{i=1}^{2} \sum_{j=1}^{2} \partial_{i}\left(k_{i j}^{(m)} \partial_{j} T_{k}^{(m)}+B_{i j k}^{(m)}\right)+\varepsilon \sum_{i=1}^{2} \partial_{i}\left(k_{i 3}^{(m)} \partial_{3} T_{k}^{(m)}+B_{i 3 k}^{(m)}\right)+ \\
& \quad+\partial_{3}\left[\varepsilon \sum_{i=1}^{2}\left(k_{3 i}^{(m)} \partial_{i} T_{k}^{(m)}+B_{3 i k}^{(m)}\right)+k_{33}^{(m)} \partial_{3} T_{k}^{(m)}+B_{33 k}^{(m)}\right]+ \\
&+\varepsilon^{2} \delta_{0 k} Q^{(m)}(\mathbf{x}, t), \quad 1 \leqslant m \leqslant M
\end{aligned}
$$

$$
\begin{gathered}
\varepsilon \sum_{i=1}^{2}\left(k_{3 i}^{(m)} \partial_{i} T_{k}^{(m)}+B_{3 i k}^{(m)}\right)+k_{33}^{(m)} \partial_{3} T_{k}^{(m)}+B_{33 k}^{(m)}= \\
=\varepsilon \sum_{i=1}^{2}\left(k_{3 i}^{(n)} \partial_{i} T_{k}^{(n)}+B_{3 i k}^{(n)}\right)+k_{33}^{(n)} \partial_{3} T_{k}^{(n)}+B_{33 k}^{(n)}, \\
T_{k}^{(m)}=T_{k}^{(n)}, \quad x_{3}=H_{m}, n=m+1,1 \leqslant m \leqslant M-1 ;
\end{gathered}
$$

$$
\begin{aligned}
& \beta^{(-)}\left[\varepsilon \sum_{i=1}^{2}\left(k_{3 i}^{(1)} \partial_{i} T_{k}^{(1)}+B_{3 i k}^{(1)}\right)\right.\left.+k_{33}^{(1)} \partial_{3} T_{k}^{(1)}+B_{33 k}^{(1)}\right]-\varepsilon \delta^{(-)} \alpha_{(-)} T_{k}^{(1)}= \\
&=\varepsilon \delta_{0 k}\left(\gamma^{(-)} Q^{(-)}-\delta^{(-)} \alpha_{(-)} T_{\infty}^{(-)}\right), \quad x_{3}=0 ;
\end{aligned}
$$

$$
\begin{aligned}
-\beta^{(+)}[\varepsilon & \left.\sum_{i=1}^{2}\left(k_{3 i}^{(M)} \partial_{i} T_{k}^{(M)}+B_{3 i k}^{(M)}\right)+k_{33}^{(M)} \partial_{3} T_{k}^{(M)}+B_{33 k}^{(M)}\right]- \\
& \quad-\varepsilon \delta^{(+)} \alpha_{(+)} T_{k}^{(M)}=\varepsilon \delta_{0 k}\left(\gamma^{(+)} Q^{(+)}-\delta^{(+)} \alpha_{(+)} T_{\infty}^{(+)}\right), \quad x_{3}=H ;
\end{aligned}
$$

$$
\begin{gathered}
-\beta \varepsilon \sum_{i=1}^{3} \sum_{j=1}^{2} n_{i}\left(k_{i j}^{(m)} \partial_{j} T_{k}^{(m)}+B_{i j k}^{(m)}\right)-\beta \sum_{i=1}^{3} n_{i}\left(k_{i 3}^{(m)} \partial_{3} T_{k}^{(m)}+B_{i 3 k}^{(m)}\right)- \\
-\varepsilon \delta \alpha^{(m)} T_{k}^{(m)}=\varepsilon \delta_{0 k}\left(\gamma q_{n}^{(m)}-\delta \alpha^{(m)} T_{\infty}\right), \quad \mathbf{x} \in S, t \geqslant t_{0}, \quad 1 \leqslant m \leqslant M ; \\
T_{k}^{(m)}\left(\mathbf{x}, t_{0}\right)=\delta_{0 k} T_{000}^{(m)}(\mathbf{x}), \quad 1 \leqslant m \leqslant M, \quad k \geqslant 0,
\end{gathered}
$$

где

$$
\begin{aligned}
C_{k}^{(m)}(\mathbf{x}, t) & \equiv \frac{1}{2} C_{* *}^{(m)}(\mathbf{x}) \sum_{l=0}^{k-1} \partial_{t}\left(T_{l}^{(m)} T_{k-l-1}^{(m)}\right), \\
B_{i j k}^{(m)}(\mathbf{x}, t) & \equiv \frac{1}{2} \beta_{i j}^{(m)}(\mathbf{x}) \sum_{l=0}^{k-1} \partial_{j}\left(T_{l}^{(m)} T_{k-l-1}^{(m)}\right), \\
1 \leqslant m & \leqslant M, \quad i, j=\overline{1,3}, \quad k \geqslant 0 ;
\end{aligned}
$$


$\delta_{0 k}$ - символ Кронекера. Согласно (17), в (24) нужно учесть, что

$$
T_{-1}^{(m)} \equiv 0, \quad 1 \leqslant m \leqslant M
$$

На основании $(24),(25)$ при $k=0$ в соотношениях (18)-(24) имеем

$$
C_{0}^{(m)} \equiv 0, \quad B_{i j 0}^{(m)} \equiv 0, \quad 1 \leqslant m \leqslant M, \quad i, j=\overline{1,3} .
$$

Если функции $T_{n}^{(m)}(0 \leqslant n \leqslant k-1)$ уже последовательно определены из начально-краевых задач (18)-(23), то согласно $(24),(26)$ функции $C_{k}^{(m)}, B_{i j k}^{(m)}$ в (18)-(22) известны. Следовательно, для каждого $k \geqslant 0$ начально-краевая задача (18)-(23) является линейной задачей нестационарной теплопроводности.

Линеаризованные уравнения (18)-(23) справедливы для граничных условий любого рода на лицевых поверхностях (20), (21). Далее предполагаем, что на этих поверхностях заданы граничные условия I рода, т.е. в (20), (21) следует принять $\beta^{( \pm)}=0, \gamma^{( \pm)}=0$, поэтому получаем

$$
T_{k}^{(1)}=\delta_{0 k} T_{\infty}^{(-)} \quad\left(x_{3}=0\right), \quad T_{k}^{(M)}=\delta_{0 k} T_{\infty}^{(+)}, \quad x_{3}=H, k \geqslant 0 .
$$

Наличие малого геометрического параметра $\varepsilon$ при высших производных в уравнении (18) в условиях сопряжения (19) и граничном условии (22) указывает на то, что при каждом $k \geqslant 0$ начально-краевая задача (18), (19), (22), (23), (27) является задачей с сингулярным возмущением, поэтому решение этой задачи следует разыскивать в виде

$$
T_{k}^{(m)}=T_{* k}^{(m)}+T_{\tau k}^{(m)}+T_{b k}^{(m)}, \quad 1 \leqslant m \leqslant M, k=0,1,2,3, \ldots,
$$

где $T_{* k}^{(m)}$ - внешнее асимптотическое разложение функции $T_{k}^{(m)}$, характеризующее основное температурное поле в $m$-том слое; $T_{\tau k}^{(m)}-$ поправка к внешнему разложению в окрестности начального момента времени $t=t_{0} ; T_{b k}^{(m)}-$ поправка к внешнему разложению в пограничном слое в окрестности торцевой поверхности оболочки (пластины).

Далее настоящее исследование посвящено определению внешнего асимптотического разложения $T_{* k}^{(m)}$. Чтобы получить для определения $T_{* k}^{(m)}$ непротиворечивую цепочку равенств, асимптотическое разложение следует задать в виде

$$
T_{* k}^{(m)}(\mathbf{x}, t) \sim \sum_{s=0}^{\infty} T_{k s}^{(m)}(\mathbf{x}, t) \varepsilon^{s}, \quad 1 \leqslant m \leqslant M, k \geqslant 0 .
$$

Подставим (29) в (18), (19), (22), (23), (27) и соберём слагаемые при одинаковых степенях $\varepsilon$, тогда получим следующую цепочку равенств для определения функций $T_{k s}^{(m)}(\mathbf{x}, t)$ :

$$
\partial_{3}\left(k_{33}^{(m)} \partial_{3} T_{k s}^{(m)}+\sum_{p=1}^{2} k_{3 p}^{(m)} \partial_{p} T_{k s-1}^{(m)}+q_{k s}^{(m)}(\mathbf{x}, t)\right)+
$$




$$
\begin{gathered}
+\sum_{p=1}^{2} \partial_{p}\left(k_{p 3}^{(m)} \partial_{3} T_{k s-1}^{(m)}\right)+\sum_{p=1}^{2} \sum_{r=1}^{2} \partial_{p}\left(k_{p r}^{(m)} \partial_{r} T_{k s-2}^{(m)}\right)- \\
-C_{*}^{(m)} \partial_{t} T_{k s-2}^{(m)}=-w_{k s}^{(m)}(\mathbf{x}, t), \quad 1 \leqslant m \leqslant M ; \\
k_{33}^{(m)} \partial_{3} T_{k s}^{(m)}+\sum_{p=1}^{2} k_{3 p}^{(m)} \partial_{p} T_{k s-1}^{(m)}+q_{k s}^{(m)}(\mathbf{x}, t)=k_{33}^{(n)} \partial_{3} T_{k s}^{(n)}+ \\
+\sum_{p=1}^{2} k_{3 p}^{(n)} \partial_{p} T_{k s-1}^{(n)}+q_{k s}^{(n)}(\mathbf{x}, t), \quad T_{k s}^{(m)}=T_{k s}^{(n)}, \\
x_{3}=H_{m}, n=m+1,1 \leqslant m \leqslant M-1 ; \\
T_{k s}^{(1)}=\delta_{0 k} \delta_{0 s} T_{\infty}^{(-)} \quad x_{3}=0 ; \quad T_{k s}^{(M)}=\delta_{0 k} \delta_{0 s} T_{\infty}^{(+)}, \quad x_{3}=H ; \\
3 \sum_{p=1}^{2} \sum_{r=1}^{2} n_{p} k_{p r}^{(m)} \partial_{r} T_{k s-1}^{(m)}-\beta \sum_{p=1}^{3} n_{p} k_{p 3}^{(m)} \partial_{3} T_{k s}^{(m)}- \\
-\delta \alpha^{(m)} T_{k s-1}^{(m)}=Q_{k s}^{(m)}(\mathbf{x}, t), \quad \mathbf{x} \in S, t \geqslant t_{0}, 1 \leqslant m \leqslant M ; \\
T_{k s}^{(m)}\left(\mathbf{x}, t_{0}\right)=\delta_{0 k} \delta_{0 s} T_{000}^{(m)}(\mathbf{x}), \quad 1 \leqslant m \leqslant M, k, s=0,1,2,3, \ldots
\end{gathered}
$$

где

$$
\begin{aligned}
& q_{k s}^{(m)}(\mathbf{x}, t) \equiv \delta_{0 s} B_{33 k}^{(m)}+\delta_{1 s} \sum_{p=1}^{2} B_{3 p k}^{(m)} \\
& w_{k s}^{(m)}(\mathbf{x}, t) \equiv \delta_{2 s}\left(\delta_{0 k} Q^{(m)}-C_{k}^{(m)}\right)+\sum_{p=1}^{2}\left(\delta_{2 s} \sum_{r=1}^{2} \partial_{p} B_{p r k}^{(m)}+\delta_{1 s} \partial_{p} B_{p 3 k}^{(m)}\right) \\
& Q_{k s}^{(m)}(\mathbf{x}, t) \equiv \delta_{0 k} \delta_{1 s}\left(\gamma q_{n}^{(m)}-\delta \alpha^{(m)} T_{\infty}\right)+\beta \delta_{0 s} \sum_{p=1}^{3} n_{p} B_{p 3 k}^{(m)}+\beta \delta_{1 s} \sum_{p=1}^{3} \sum_{r=1}^{2} n_{p} B_{p r k}^{(m)}
\end{aligned}
$$

Согласно (29), в (30)-(33) нужно учесть, что

$$
T_{k,-1}^{(m)} \equiv 0, \quad T_{k,-2}^{(m)} \equiv 0, \quad 1 \leqslant m \leqslant M, k=0,1,2,3, \ldots
$$

В силу равенств $(35)$ функции $q_{k s}^{(m)}, w_{k s}^{(m)}, Q_{k s}^{(m)}$ в $(30),(31),(33)$ известны при каждом $s \geqslant 0$.

Проинтегрировав уравнение (30) по переменной $x_{3}$ при $s=0$ с учётом (31), (35), (36), получим

$$
k_{33}^{(m)} \partial_{3} T_{k 0}^{(m)}+q_{k 0}^{(m)}(\mathbf{x}, t)=Q_{k 0}^{0}\left(x_{1}, x_{2}, t\right), \quad 1 \leqslant m \leqslant M
$$

откуда

$$
\partial_{3} T_{k 0}^{(m)}=\frac{1}{k_{33}^{(m)}(\mathbf{x})}\left(Q_{k 0}^{0}\left(x_{1}, x_{2}, t\right)-q_{k 0}^{(m)}(\mathbf{x}, t)\right), \quad 1 \leqslant m \leqslant M, k \geqslant 0,
$$


где $Q_{k 0}^{0}\left(x_{1}, x_{2}, t\right)$ - произвольная функция, подлежащая в последующем определению.

Проинтегрируем (37) с учётом второго равенства (31) и граничных условий (32), тогда будем иметь

$$
\begin{aligned}
& T_{k 0}^{(m)}(\mathbf{x}, t)= \delta_{0 k} T_{\infty}^{(-)}\left(x_{1}, x_{2}, t\right)+ \\
&+ Q_{k 0}^{0}\left(x_{1}, x_{2}, t\right)\left(\int_{H_{m-1}}^{x_{3}} \frac{d x_{3}}{k_{33}^{(m)}(\mathbf{x})}+\sum_{l=1}^{m-1} \int_{H_{l-1}}^{H_{l}} \frac{d x_{3}}{k_{33}^{(l)}(\mathbf{x})}\right)- \\
&-\int_{H_{m-1}}^{x_{3}} \frac{q_{k 0}^{(m)}(\mathbf{x}, t)}{k_{33}^{(m)}(\mathbf{x})} d x_{3}-\sum_{l=1}^{m-1} \int_{H_{l-1}}^{H_{l}} \frac{q_{k 0}^{(l)}(\mathbf{x}, t)}{k_{33}^{(l)}(\mathbf{x})} d x_{3}, \\
& H_{m-1}<x_{3} \leqslant H_{m}, \quad 1 \leqslant m \leqslant M ;
\end{aligned}
$$

$$
\begin{aligned}
Q_{k 0}^{0}\left(x_{1}, x_{2}, t\right)= & \left(\sum_{m=1}^{M}\right. \\
& \left.\int_{H_{m-1}}^{H_{m}} \frac{d x_{3}}{k_{33}^{(m)}(\mathbf{x})}\right)^{-1} \times \\
& \times\left(\delta_{0 k} T_{\infty}^{(+)}-\delta_{0 k} T_{\infty}^{(-)}+\sum_{m=1}^{M} \int_{H_{m-1}}^{H_{m}} \frac{q_{k 0}^{(m)}(\mathbf{x}, t)}{k_{33}^{(m)}(\mathbf{x})} d x_{3}\right) .
\end{aligned}
$$

Функция $T_{k 0}^{(m)}(\mathbf{x}, t)$ полностью определяется конечными соотношениями (38), (39), т. е. граничными условиями на лицевых поверхностях.

Граничное условие на кромке (33) и начальное условие (34) при $s=0$ с учётом (36), (37)-(39) могут быть выполнены тождественно как в локальном, так и в интегральном (после интегрирования по толщине оболочки или пластины) смысле лишь в исключительных случаях. В общем случае граничное условие на кромке тонкостенной конструкции можно удовлетворить только после рассмотрения пограничных слоёв (после определения поправки $T_{b k}^{(m)}$ в $\left.(28)\right)$, а начальное условие можно выполнить лишь после определения в окрестности начального момента времени $t=t_{0}$ поправки $T_{\tau k}^{(m)}$ в разложении (28).

Проинтегрируем уравнение (30) по переменной $x_{3}$ при $s=1$ с учётом (31), (35)-(37), тогда получим

$$
\begin{aligned}
k_{33}^{(m)} \partial_{3} T_{k 1}^{(m)}+\sum_{p=1}^{2} k_{3 p}^{(m)} \partial_{p} T_{k 0}^{(m)} & +q_{k 1}^{(m)}(\mathbf{x}, t)= \\
& =Q_{k 1}^{0}\left(x_{1}, x_{2}, t\right)-\Phi_{k 1}^{(m)}(\mathbf{x}, t), \quad 1 \leqslant m \leqslant M,
\end{aligned}
$$

где

$$
\begin{aligned}
& \Phi_{k 1}^{(m)}(\mathbf{x}, t) \equiv \int_{H_{m-1}}^{x_{3}} W_{k 1}^{(m)}(\mathbf{x}, t) d x_{3}+\sum_{l=1}^{m-1} \int_{H_{l-1}}^{H_{l}} W_{k 1}^{(l)}(\mathbf{x}, t) d x_{3}, H_{m-1}<x_{3} \leqslant H_{m} \\
& W_{k 1}^{(m)}(\mathbf{x}, t) \equiv w_{k 1}^{(m)}(\mathbf{x}, t)+\sum_{p=1}^{2} \partial_{p}\left[\frac{k_{p 3}^{(m)}}{k_{33}^{(m)}}\left(Q_{k 0}^{0}\left(x_{1}, x_{2}, t\right)-q_{k 0}^{(m)}(\mathbf{x}, t)\right)\right]
\end{aligned}
$$


$Q_{k 1}^{0}\left(x_{1}, x_{2}, t\right)$ - произвольная функция, подлежащая определению. Согласно (35), (39), (41) функция $\Phi_{k 1}^{(m)}$ в $(40)$ известна.

Выразим из (40) производную

$$
\partial_{3} T_{k 1}^{(m)}=\frac{Q_{k 1}^{0}\left(x_{1}, x_{2}, t\right)}{k_{33}^{(m)}(\mathbf{x})}-\Theta_{k 1}^{(m)}(\mathbf{x}, t), \quad 1 \leqslant m \leqslant M,
$$

где согласно $(38),(39),(41),(35)$ известная функция $\Theta_{k 1}^{(m)}$ определяется так:

$$
\Theta_{k 1}^{(m)}(\mathbf{x}, t) \equiv \frac{1}{k_{33}^{(m)}(\mathbf{x})}\left[\Phi_{k 1}^{(m)}(\mathbf{x}, t)+\sum_{p=1}^{2} k_{3 p}^{(m)} \partial_{p} T_{k 0}^{(m)}+q_{k 1}^{(m)}(\mathbf{x}, t)\right] .
$$

Проинтегрируем (42) по $x_{3}$ с учётом $(43),(31),(32)$, тогда будем иметь

$$
\begin{aligned}
& T_{k 1}^{(m)}(\mathbf{x}, t)=Q_{k 1}^{0}\left(x_{1}, x_{2}, t\right)\left(\int_{H_{m-1}}^{x_{3}} \frac{d x_{3}}{k_{33}^{(m)}(\mathbf{x})}+\sum_{l=1}^{m-1} \int_{H_{l-1}}^{H_{l}} \frac{d x_{3}}{k_{33}^{(l)}(\mathbf{x})}\right)- \\
&-\Psi_{k 1}^{(m)}(\mathbf{x}, t), \quad H_{m-1}<x_{3} \leqslant H_{m}, 1 \leqslant m \leqslant M,
\end{aligned}
$$

где известная функция $\Psi_{k 1}^{(m)}$ задаётся выражением

$$
\begin{aligned}
\Psi_{k 1}^{(m)}(\mathbf{x}, t) \equiv \int_{H_{m-1}}^{x_{3}} \Theta_{k 1}^{(m)}(\mathbf{x}, t) d x_{3}+ & \\
& +\sum_{l=1}^{m-1} \int_{H_{l-1}}^{H_{l}} \Theta_{k 1}^{(l)}(\mathbf{x}, t) d x_{3}, \quad H_{m-1}<x_{3} \leqslant H_{m} .
\end{aligned}
$$

Подставляя (44) во второе граничное условие (32) при $s=1$, получим

$$
Q_{k 1}^{0}\left(x_{1}, x_{2}, t\right)=\left.\left(\sum_{m=1}^{M} \int_{H_{m-1}}^{H_{m}} \frac{d x_{3}}{k_{33}^{(m)}(\mathbf{x})}\right)^{-1} \Psi_{k 1}^{(M)}(\mathbf{x}, t)\right|_{x_{3}=H} .
$$

Следовательно, конечные соотношения (44), (46) с учётом (45), (43), (41), (35) полностью определяют коэффициент $T_{k 1}^{(m)}(\mathbf{x}, t)$ асимптотического ряда (29). Как и при $s=0$, граничное условие на кромке (33) и начальное условие $(34)$ при $s=1$ могут быть выполнены лишь после получения поправок $T_{b k}^{(m)}$ и $T_{\tau k}^{(m)}$ в разложении $(28)$.

Далее предполагаем, что при $s \geqslant 2$ функции $T_{k s-2}^{(m)}(\mathbf{x}, t), T_{k s-1}^{(m)}(\mathbf{x}, t)$ уже известны и справедливо выражение для производной

$$
\partial_{3} T_{k s-1}^{(m)}=\frac{Q_{k s-1}^{0}\left(x_{1}, x_{2}, t\right)}{k_{33}^{(m)}(\mathbf{x})}-\Theta_{k s-1}^{(m)}(\mathbf{x}, t), \quad 1 \leqslant m \leqslant M, s \geqslant 2,
$$

где $Q_{k s-1}^{0}, \Theta_{k s-1}^{(m)}$ - также уже известные функции. (В силу (42)-(46), (38), (39) эти предположения справедливы при $s=2$. ) 
Проинтегрируем уравнение (30) по переменной $x_{3}$ при $s \geqslant 2$ с учётом (31), (35), (47) и сделанных предположений, тогда получим

$$
\begin{aligned}
& k_{33}^{(m)} \partial_{3} T_{k s}^{(m)}+\sum_{p=1}^{2} k_{3 p}^{(m)} \partial_{p} T_{k s-1}^{(m)}= \\
& \quad=Q_{k s}^{0}\left(x_{1}, x_{2}, t\right)-\Phi_{k s}^{(m)}(\mathbf{x}, t), \quad 1 \leqslant m \leqslant M, s \geqslant 2,
\end{aligned}
$$

где

$$
\begin{gathered}
\Phi_{k s}^{(m)}(\mathbf{x}, t) \equiv \int_{H_{m-1}}^{x_{3}} W_{k s}^{(m)}(\mathbf{x}, t) d x_{3}+ \\
\quad+\sum_{l=1}^{m-1} \int_{H_{l-1}}^{H_{l}} W_{k s}^{(l)}(\mathbf{x}, t) d x_{3}, \quad H_{m-1}<x_{3} \leqslant H_{m}, \\
W_{k s}^{(m)}(\mathbf{x}, t) \equiv w_{k s}^{(m)}(\mathbf{x}, t)-C_{*}^{(m)} \partial_{t} T_{k s-2}^{(m)}+\sum_{p=1}^{2} \sum_{r=1}^{2} \partial_{p}\left(k_{p r}^{(m)} \partial_{r} T_{k s-2}^{(m)}\right)+ \\
+\sum_{p=1}^{2} \partial_{p}\left[\frac{k_{p 3}^{(m)}}{k_{33}^{(m)}}\left(Q_{k s-1}^{0}\left(x_{1}, x_{2}, t\right)-k_{33}^{(m)} \Theta_{k s-1}^{(m)}(\mathbf{x}, t)\right)\right], \quad 1 \leqslant m \leqslant M ;
\end{gathered}
$$

$Q_{k s}^{0}\left(x_{1}, x_{2}, t\right)$ - произвольная функция, подлежащая определению. Согласно $(49),(47),(35)$ и принятым предположениям, функция $\Phi_{k s}^{(m)}$ в $(48)$ известна.

Выразим из (48) производную

$$
\partial_{3} T_{k s}^{(m)}=\frac{Q_{k s}^{0}\left(x_{1}, x_{2}, t\right)}{k_{33}^{(m)}(\mathbf{x})}-\Theta_{k s}^{(m)}(\mathbf{x}, t), \quad 1 \leqslant m \leqslant M, s \geqslant 2,
$$

где согласно (48) и сделанным предположениям известная функция $\Theta_{k s}^{(m)}$ определяется так:

$$
\Theta_{k s}^{(m)}(\mathbf{x}, t) \equiv \frac{1}{k_{33}^{(m)}(\mathbf{x})}\left[\Phi_{k s}^{(m)}(\mathbf{x}, t)+\sum_{p=1}^{2} k_{3 p}^{(m)} \partial_{p} T_{k s-1}^{(m)}\right], \quad 1 \leqslant m \leqslant M, s \geqslant 2 .
$$
иметь

Проинтегрируем (50) по $x_{3}$ с учетом $(51),(31),(32)$ при $s \geqslant 2$, тогда будем

$$
\begin{aligned}
& T_{k s}^{(m)}(\mathbf{x}, t)=Q_{k s}^{0}\left(x_{1}, x_{2}, t\right)\left(\int_{H_{m-1}}^{x_{3}} \frac{d x_{3}}{k_{33}^{(m)}(\mathbf{x})}+\sum_{l=1}^{m-1} \int_{H_{l-1}}^{H_{l}} \frac{d x_{3}}{k_{33}^{(l)}(\mathbf{x})}\right)- \\
&-\Psi_{k s}^{(m)}(\mathbf{x}, t), \quad H_{m-1}<x_{3} \leqslant H_{m}, 1 \leqslant m \leqslant M, s \geqslant 2,
\end{aligned}
$$

где известная функция $\Psi_{k s}^{(m)}$ задаётся выражением 


$$
\begin{aligned}
\Psi_{k s}^{(m)}(\mathbf{x}, t) \equiv \int_{H_{m-1}}^{x_{3}} & \Theta_{k s}^{(m)}(\mathbf{x}, t) d x_{3}+ \\
& +\sum_{l=1}^{m-1} \int_{H_{l-1}}^{H_{l}} \Theta_{k s}^{(l)}(\mathbf{x}, t) d x_{3}, \quad H_{m-1}<x_{3} \leqslant H_{m}, s \geqslant 2 .
\end{aligned}
$$

Подставляя (52) во второе граничное условие (32) при $s \geqslant 2$, получим

$$
Q_{k s}^{0}\left(x_{1}, x_{2}, t\right)=\left.\left(\sum_{m=1}^{M} \int_{H_{m-1}}^{H_{m}} \frac{d x_{3}}{k_{33}^{(m)}(\mathbf{x})}\right)^{-1} \Psi_{k s}^{(M)}(\mathbf{x}, t)\right|_{x_{3}=H}, s \geqslant 2, \quad k \geqslant 0 .
$$

Таким образом, конечные соотношения (52), (54) с учётом (53), (51), (49), (35) полностью определяют коэффициент $T_{k s}^{(m)}(\mathbf{x}, t)$ асимптотического ряда (29) при $s \geqslant 2, k \geqslant 0$. Как и при $s=0, s=1$, граничное условие на кромке (33) и начальное условие (34) при $s \geqslant 2$ могут быть выполнены лишь после получения поправок $T_{b k}^{(m)}$ и $T_{\tau k}^{(m)}$ в разложении $(28)$.

Так как соотношения $(47),(50)$ формально совпадают и функции $\Theta_{k s}^{(m)}(\mathbf{x}, t)$, $T_{k s}^{(m)}(\mathbf{x}, t), T_{k s-1}^{(m)}(\mathbf{x}, t)$ уже известны, принятые выше предположения становятся справедливыми при новом значении $s$ (при замене в $(47)(s-1)$ на $s$ ), поэтому по схеме (47)-(54) при $s \geqslant 2$ можно последовательно определить все коэффициенты $T_{k s}^{(m)}(\mathbf{x}, t)$ асимптотического ряда $(29)$.

Из соотношений (38), (39), (52), (54) с учётом (35) следует, что в случае задания на лицевых поверхностях граничных условий I рода температуры этих поверхностей $T_{\infty}^{(-)}, T_{\infty}^{(+)}$оказывают на температуру оболочки (или пластины) на два порядка по $\varepsilon$ большее влияние, чем внутренние источники тепла, так как температуры $T_{\infty}^{(-)}, T_{\infty}^{(+)}$при $k=0$ определяют функцию $T_{00}^{(m)}$ (см. (38), (39), (35)) и все последующие $T_{k s}^{(m)}(k \geqslant 0, s \geqslant 1)$, а плотность мощности внутренних источников тепла $Q^{(m)}$ определяет $T_{02}^{(m)}$ (см. (52)-(54) с учётом (51), (49), (35) при $k=0, s=2)$ и все последующие функции $T_{k s}^{(m)}(k \geqslant 0$, $s \geqslant 3)$.

Из равенств (38)-(54) с учётом (35) следует, что при однородности материалов слоёв по толщине пластины $\left(\partial_{3} k_{i j}^{(m)}=0\right)$ в линейном приближении (без учета термочувствительности: $\beta_{i j}^{(m)}=0$ ) с точностью $O(\varepsilon)$ распределение температуры в каждом слое в поперечном направлении можно задавать по линейному закону (по кусочно-линейному закону для всего пакета); с точностью же $O\left(\varepsilon^{2}\right)$ температуру по толщине слоёв пластины можно задавать по квадратичному закону (по кусочно-квадратичному закону для всей пластины). Этот вывод в общем случае не относится к оболочкам, так как для них согласно $(15) \partial_{3} k_{i j}^{(m)} \neq 0, i, j=\overline{1,3}$.

Из соотношений (38), (39) вытекает, что при задании на обеих лицевых поверхностях одинаковой температуры $T_{\infty}^{(+)}=T_{\infty}^{(-)}$получим $T_{00}^{(m)}=T_{\infty}^{(+)}=$ $=T_{\infty}^{(-)}, Q_{00}^{0} \equiv 0$, т. е. с точностью $O(\varepsilon)$ можно температуру считать постоянной по толщине слоистой оболочки (пластины); если же, кроме того, $T_{\infty}^{(+)}=T_{\infty}^{(-)}=\mathrm{const}$, то, согласно $(38)-(46)$, получим $T_{01}^{(m)}=0$ и температуру 
по толщине слоистой тонкостенной конструкции за пределами пограничного слоя можно считать постоянной с точностью $O\left(\varepsilon^{2}\right)$; если же отсутствуют и внутренние источники тепла $\left(Q^{(m)} \equiv 0\right)$, то из $(52)-(54)$ с учетом $(49),(51)$ дополнительно получим $T_{0 i}^{(m)} \equiv 0, T_{k j}^{(m)} \equiv 0(k \geqslant 1, i \geqslant 2, j \geqslant 0)$, т. е. в этом случае за пределами пограничного слоя температура в оболочке или пластине постоянна $\left(T_{*}^{(m)}=T_{00}^{(m)}=T_{\infty}^{(+)}=T_{\infty}^{(-)}=\right.$const $)$.

Как уже отмечалось, построенные выше внешние асимптотические разложения температуры могут привести к невязкам в граничных условиях (13) на кромках оболочки или пластины [17] и в начальных условиях (14), для устранения которых можно использовать обычную процедуру $[16,17]$ введения в окрестности кромки и начального момента времени $t=t_{0}$ внутренних «растянутых» переменных и построения соответствующих внутренних асимптотических разложений типа «пограничных слоёв» с последующим «сшиванием» (согласованием) их с внешним разложением.

Если особый интерес вызывает поведение температурного поля именно в окрестности начального момента времени $t=t_{0}$, то целесообразно сразу вводить растянутый масштаб времени. В этом случае при обезразмеривании величин $\bar{c}^{(m)} \bar{\rho}^{(m)}$ вместо формул (15) нужно использовать

$$
\begin{aligned}
& C^{(m)}=A_{1} A_{2} A_{3} \bar{H}_{*}^{2} \bar{c}^{(m)} \bar{\rho}^{(m)} /\left(\bar{\lambda}_{*} \bar{t}_{*}\right), \quad C_{*}^{(m)}=A_{1} A_{2} A_{3} \bar{H}_{*}^{2} \bar{c}_{*}^{(m)} \bar{\rho}^{(m)} /\left(\bar{\lambda}_{*} \bar{t}_{*}\right), \\
& \eta_{*} C_{* *}^{(m)}=A_{1} A_{2} A_{3} \bar{H}_{*}^{2} \bar{T}_{*} \bar{c}_{* *}^{(m)} \bar{\rho}^{(m)} /\left(\bar{\lambda}_{*} \bar{t}_{*}\right), \quad 1 \leqslant m \leqslant M,
\end{aligned}
$$

причём характерное время $\bar{t}_{*}$, в отличие от предыдущего, нужно выбирать так, чтобы $\bar{H}_{*}^{2} / \bar{t}_{*}=$ const и величины $C_{*}^{(m)}, \eta_{*} C_{* *}^{(m)}$ имели значения порядка единицы. При таком выборе значения $\bar{t}_{*}$ согласно (55) справедливыми остаются асимптотические оценки (16), а в левой части уравнения (8) не будет присутствовать малый сомножитель $\varepsilon^{2}$, поэтому вместо уравнения (30) при $k=s=0$ с учётом (35), (36) получим

$$
C_{*}^{(m)} \partial_{t} T_{00}^{(m)}=\partial_{3}\left(k_{33}^{(m)} \partial_{3} T_{00}^{(m)}\right), \quad 1 \leqslant m \leqslant M .
$$

Подобную структуру будут иметь уравнения, аналогичные (30), и при $k \geqslant 0, s \geqslant 0$ (при этом в (30) нужно формально $\partial_{t} T_{k s-2}^{(m)}$ заменить на $\partial_{t} T_{k s}^{(m)}$ ). Уравнение (56) является линейным уравнением параболического типа. В общем случае, когда $k_{33}^{(m)}$ зависит от переменной $x_{3}$ (например, при рассмотрении оболочек), это уравнение можно проинтегрировать только численно [18]. В случае пластин с однородными по толщине слоями $\left(\partial_{3} k_{33}^{(m)} \equiv 0\right)$ можно получить решение уравнения (56) в аналитической форме (либо точно, либо приближенно [13]). Изучение этих вопросов выходит за рамки настоящего исследования.

Выше рассматривалась задача теплопроводности тонкостенных конструкций при задании на обеих лицевых поверхностях граничных условиях I рода, которые являются предельным случаем граничных условий III рода при бесконечно больших числах Био $\left(\alpha_{( \pm)} \rightarrow \infty\right.$, см. (11), (12), (20) (21)). Если хотя бы на одной из лицевых поверхностей заданы граничные условия III рода с конечным значением числа Био, то, как показывает [11], можно построить внешнее асимптотическое разложение температурного поля без 
предварительной линеаризации задачи теплопроводности (без использования разложения (17)). Кроме того, отдельного рассмотрения требует случай конвективного теплообмена на обеих лицевых поверхностях с малыми числами Био $\left(\alpha_{( \pm)}<1\right)$. Изучение этих вопросов также выходит за рамки настоящего исследования.

Следует отметить, что в стационарном случае для слоистой пластины, слои которой однородны по толщине $\left(\partial_{3} k_{33}^{(m)} \equiv 0, \partial_{3} \beta_{33}^{(m)} \equiv 0\right)$, при отсутствии внутренних источников тепла $\left(Q^{(m)} \equiv 0\right)$ и задании граничных условий I рода на лицевых поверхностях пластины решение нелинейной задачи теплопроводности (без предварительной её линеаризации) в аналитической форме приведено в [13].

Заключение. В теоретических и прикладных исследованиях часто приходится решать задачи термоупругости, термопластичности и аналогичные, при этом, если постановка задачи несвязная, то решение задачи теплопроводности предшествует решению о напряжённо-деформированном состоянии. Полученное в настоящей работе внешнее асимптотическое разложение решения нелинейной задачи теплопроводности описывает, по сути, квазистационарное тепловое состояние тонкостенной слоистой анизотропной неоднородной конструкции при учете термочувствительности материалов слоёв. Поэтому оно может быть использовано, например, при расчётах композитных оболочек и пластин на ползучесть и длительную прочность, так как при таких расчётах рассматриваются большие временные интервалы (значение характерного времени $\bar{t}_{*}$ велико, см. (15)), на которых динамические и сильно выраженные нестационарные эффекты считаются уже затухшими. Известно, что механическое поведение материалов в условиях температурной ползучести существенно зависит от температуры [19], поэтому при решении краевых задач расчёта напряжённо-деформированного состояния тонкостенных (и других) конструкций температурное поле играет определяющую роль. Для более точного определения температуры необходимо учитывать термочувствительность материалов слоёв таких конструкций, что и было проделано в настоящем исследовании. Допустимость полученного решения задачи теплопроводности в практических расчетах на прочность можно обосновать еще и тем, что в инженерной практике, как правило, используются приближенные теории изгиба оболочек и пластин (Кирхгофа-Лява, Тимошенко-Рейснера и др. [20-22]), которые дают приемлемую точность лишь на некотором удалении от кромок тонкостенных конструкций, т. е. за пределами пограничных слоёв и локальных эффектов, распространяющихся в глубину конструкции на расстояние порядка её толщины [20,21].

Работа выполнена при поддержке РФФИ (проект № 14-01-90400-Укр_а).

This work is supported by RFBR, project no. 14-01-90400-Ukr_a.

\section{СПИСОК ЛИТЕРАТУРЫ/ REFERENCES}

1. Ю. М. Тарнопольский, И. Г. Жигун, В. А. Поляков, Пространственно-армированные композиционные материалы: Справочник, М.: Машиностроение, 1987. 224 с. [Yu. M. Tarnopol'skiy, I. G. Zhigun, V. A. Polyakov, Prostranstvenno-armirovannye kompozitsionnye materialy [Space-reinforced composite materials], Moscow, Mashinostroenie, 1987, 224 pp. (In Russian)]

2. Ю. М. Тарнапольский, А. В. Розе, И. Г. Жииун, Г. М. Гуняев, "Конструкционные 
особенности материалов, армированных высокомодульными волокнами" // Механика полимеров, 1971. № 4. C. 676-685; Yu. M. Tarnopol'skii, A. V. Roze, I. G. Zhigun, G. M. Gunyaev, "Structural characteristics of materials reinforced with high-modulus fibers", Polymer Mechanics, 1971, vol. 7, no. 4, pp. 600-609. doi: 10.1007/BF00855201.

3. Д. В. Дедков, А. А. Ташкинов, "Коэффициенты концентрации напряжений в слое тканого композита с локальными технологическими дефектами при чистом формоизменении" // Вычислительная механика сплошных сред, 2013. №6. С. 103-109 doi: 10 . 7242/1999-6691/2013.6.1.13. [D. V. Dedkov, A. A. Tashkinov, "Stress concentration coefficients of a woven textile composite layer with local processing defects under pure forming conditions", Computational Continuum Mechanics, 2013, no.6, pp. 103-109. (In Russian)].

4. M. H. Mohamed, A. E. Bogdanovich, L. C. Dickinson, J. N. Singletary, R. R. Lienhart, "A new generation of 3D woven fabric performs and composites", SAMPE Journal, 2001, vol. 37, no. 3, pp. 3-17.

5. Ю. В. Немировский, Н. А. Фёдорова, "Исследование рациональных структур криволинейного армирования в полярной системе координат" // Вестн. Сам. гос. техн. ун-та. Сер. Физ.-мат. науки, 2013. №1(30). С. 233-244. doi:10.14498/vsgtu1164. [Yu. V. Nemirovsky, N. A. Feodorova, "Study of curvilinear reinforcement rational structures in polar coordinate system", Vestn. Samar. Gos. Tekhn. Univ. Ser. Fiz.-Mat. Nauki, 2013, no. 1(30), pp. 233-244. (In Russian)].

6. Й. Шустер, Д. Гейдер, К. Шарп, М. Глования, "Измерение и моделирование теплопроводности трехмерных тканых композитов" // Механика композитных материалов, 2009. Т. 45, № 2. C. 241-254; J. Schuster, D. Heider, K. Sharp, M. Glowania, "Measuring and modeling the thermal conductivities of three-dimensionally woven fabric composites", Mechanics of Composite Materials, 2009, vol. 45, no. 2, pp. 165-174. doi: 10. 1007/s11029-009-9072-y.

7. Э. М. Карташов, В. А. Кудинов, Аналитическая теория теплопроводности и прикладной термоупругости, М.: Либроком, 2012. 656 с. [Е. M. Kartashov, V. A. Kudinov, Analiticheskaya teoriya teploprovodnosti i prikladnoy termouprugosti [Analytical Theory of Heat Conduction and Thermoelasticity], Moscow, Librokom, 2012, 656 pp. (In Russian)]

8. В. В. Болотин, Ю. Н. Новичков, Механика многослойных конструкиий, М.: Машиностроение, 1980. 375 с. [V. V. Bolotin, Yu. N. Novichkov, Mekhanika mnogosloynykh konstruktsiy [Mechanics of Multilayer Structures], Moscow, Mashinostroenie, 1980, 375 pp. (In Russian)]

9. О. В. Биткина, "Методы исследования влияния технологических погрешностей на напряженно-деформируемое состояние многослойных композитных панелей" // Известия Самарского научного центра Российской академии наук, 2012. Т. 14, № 4(2). C. 569-576. [O. V. Bitkina, "Investigation methods of the influence of technological errors on stress-strain state of multilayered composite panels", Izvestiya Samarskogo nauchnogo tsentra Rossiyskoy akademii nauk, 2012, vol. 14, no. 4(2), pp. 569-576. (In Russian)].

10. О. В. Биткина, “Экспериментальное исследование влияния технологических факторов на формоизменение многослойных панелей из композиционных материалов" // Вестн. Самар. гос. техн. ун-та. Сер. Технические науки, 2013. № 1(37). С. 99-110. [O. V. Bitkina, "Experimental investigation of the influence of technological factors on the forming of multilayered panels made of composite materials", Vestn. Samar. Gos. Tekhn. Univ. Ser. Tekhnicheskie Nauki, 2013, no.1(37), pp. 99-110. (In Russian)].

11. А. П. Янковский, “Асимптотический анализ решения нелинейной задачи нестационарной теплопроводности слоистых анизотропных неоднородных оболочек при смешанных граничных условиях на лицевых поверхностях"// Инженерно-физический журнал, 2013. Т. 86, №6. C. 1263-1273; A. P. Yankovskii, "Asymptotic Analysis of Solution of a Nonlinear Problem of Nonstationary Heat Conduction of Lamellar Anisotropic Inhomogeneous Shells with Mixed Boundary Conditions on Faces", J. Eng. Phys. Thermophys., 2013, vol.86, no.6, pp. 1344-1354. doi: 10.1007/s10891-013-0959-z. 
12. Н. М. Беляев, А. А. Рядно, Методи теории теплопроводности. Т. 1, М.: Высшая школа, 1982.327 с. [N. M. Belyaev, A. A. Ryadno, Metody teorii teploprovodnosti [Methods of Heat Conduction Theory], V.1, Moscow, Vysshaya shkola, 1982, 327 pp. (In Russian)]

13. В. А. Кудинов, И. В. Кудинов, Методы решения параболических и гиперболических уравнений теплопроводности, ред. Э. М. Карташов, М.: Либроком, 2012. 280 с. [V. A. Kudinov, I. V. Kudinov, Metody resheniya parabolicheskikh i giperbolicheskikh uravneniy teploprovodnosti [Methods for Solving Parabolic and Hyperbolic Heat Conduction Equations], ed. E. M. Kartashov, Moscow, Librokom, 2012, 280 pp. (In Russian)]

14. Б. Е. Неймарк, Физические свойства сталей и сплавов, применяемых в энергетике: Справочник, М.-Л.: Энергия, 1967. 238 с. [B. E. Neimark, Fizicheskie svoystva staley $i$ splavov, primenyaemykh $v$ energetike [Physical properties of steels and alloys in the energy sector], Moscow, Leningrad, Energiya, 1967, 238 pp. (In Russian)]

15. В. Н. Луканин, М. Г. Шатров, Г. М. Камфер, С. Г. Нечаев, И. Е. Иванов, Л. М. Матюхин, К. А. Морозов, Теплотехника, ред. В. Н. Луканин, М.: Высшая школа, 2003. 671 с. [V. N. Lukanin, M. G. Shatrov, G. M. Kamfer, S. G. Nechaev, I. E. Ivanov, L. M. Matyukhin, K. A. Morozov, Teplotekhnika [Heat Engineering], ed. V. N. Lukanin, Moscow, Vysshaya shkola, 2003, 671 pp. (In Russian)]

16. Е. И. Зино, Э. А. Тропп, Асимптотические методы в задачах теории теплопроводности и термоупругости, Л.: Ленингр. ун-т, 1978. 224 с. [E. I. Zino, E. A. Tropp, Asimptoticheskie metody $v$ zadachakh teorii teploprovodnosti $i$ termouprugosti [Asymptotic Methods in Problems of Heat Conduction and Thermoelasticity Theory], Leningrad, Leningrad State Univ. Publ., 1978, 224 pp. (In Russian)]

17. А. М. Ильин,, Согласование асимптотических разложений решений краевых задач, M.: Наука, 1989. 336 с.; A. M. Ilin, Matching of asymptotic expansions of solutions of boundary value problems, Translations of Mathematical Monographs, vol. 102, Providence, RI, American Mathematical Society, 1992, ix+279 pp.

18. Н. М. Беляев, А. А. Рядно, Методы теории теплопроводности. Т. 2, М.: Высшая школа, 1982. 304 с. [N. M. Belyaev, А. A. Ryadno, Metody teorii teploprovodnosti [Methods of Heat Conduction Theory], V.2, Moscow, Vysshaya shkola, 1982, 304 pp. (In Russian)]

19. Ю. Н. Работнов, Ползучесть элементов конструкиий, М.: Физматгиз, 1966. 752 с. [Yu. N. Rabotnov, Polzuchest' elementov konstruktsiy [Creep of Structural Elements], Moscow, Fizmatgiz, 1966, 752 pp. (In Russian)]

20. А. К. Малмейстер, В. П. Тамуж, Г. А. Тетерс, Сопротивление полимерных и композитных материалов, Рига: Зинатне, 1980. 571 с. [А. K. Malmeyster, V. P. Tamuzh, G. A. Teters, Soprotivlenie polimernykh i kompozitnykh materialov [Resistance of Polymer and Composite Matertals], Riga, Zinatne, 1980, 571 pp. (In Russian)]

21. В. В. Карпов, Прочность и устойчивость подкрепленных оболочек вращения. Т. 1: Модели и алгоритмы исследования прочности и устойчивости подкрепленных оболочек вращения, М.: Физматлит, 2010. 288 с. [V. V. Karpov, Prochnost' $i$ ustoychivost' podkreplennykh obolochek vrashcheniya [Strength and stability reinforced shells of revolution], V.1, Modeli $i$ algoritmy issledovaniya prochnosti $i$ ustoychivosti podkreplennykh obolochek vrashcheniya [Models and algorithms of strength and stability of the reinforced shells of revolution], Moscow, Fizmatlit, 2010, 288 pp. (In Russian)]

22. В. В. Карпов, А. А. Семенов, “Математическая модель деформирования подкрепленных ортотропных оболочек вращения" // Инженерно-строительный журнал, 2013. №5(40). C. 100-106. doi: 10.5862/mce.40.11. [V. V. Karpov, A. A. Semenov, "Mathematical model of deformation of orthotropic reinforced shells of revolution", Inzhenerno-stroitel'nyy zhurnal [Magazine of Civil Engineering], 2013, no.5(40), pp. 100-106. (In Russian)].

Поступила в редакцию 09/XII/2013;

в окончательном варианте - 21/II/2014; принята в печать - 19/III/2014. 
MSC: 35Q79, 80A17; 74K25

\section{ASYMPTOTIC ANALYSIS OF SOLUTIONS OF A NONLINEAR PROBLEM OF UNSTEADY HEAT CONDUCTION OF LAYERED ANISOTROPIC INHOMOGENEOUS SHELLS UNDER BOUNDARY CONDITIONS OF THE FIRST KIND ON THE FRONT SURFACES}

\section{A. P. Yankovskii}

Khristianovich Institute of Theoretical and Applied Mechanics, Siberian Branch of the Russian Academy of Sciences,

4/1, Institutskaya st., Novosibirsk, 630090, Russian Federation.

The heat conduction problem is formulated for the layered shells consisting of heatsensitive anisotropic inhomogeneous layers, with boundary conditions of general form. The heat sensitivity of the material layers is described by the linear dependence of their thermophysical characteristics on temperature. The equation of heat conduction, boundary conditions and conditions of thermal conjugations on the boundaries of the contact between the layers are written in the dimensionless form. Two small parameters in dimensionless ratios are defined: thermophysical parameter characterizing the degree of thermal sensitivity of the material layers and geometrical parameter characterizing the relative shell thickness. Sequential recursion of dimensionless ratios is carry out, first on thermophysical small parameter, and then on the geometrical parameter. The first type of recursion allowed to linearize the problem of heat conduction. On the basis of the second type of recursion the exterior asymptotic expansion of the solution is built for the problem of nonstationary heat conduction of layered anisotropic heterogeneous shells with boundary conditions of the first kind on the facial surfaces. The obtained two-dimensional governing equation is analyzed. The asymptotic properties of solutions of the problem of heat conductivity are investigated.

Keywords: thermal conductivity, thermal sensitivity, asymptotic analysis, sandwich shells, anisotropy and heterogeneity.

Received 09/XII/2013;

received in revised form $21 / \mathrm{II} / 2014$;

accepted 19/III/2014.

ISSN: 2310-7081 (online), 1991-8615 (print); doi: http://dx.doi.org/10.14498/vsgtu1281 (C) 2014 Samara State Technical University.

Citation: A. P. Yankovskii, "Asymptotic Analysis of Solutions of a Nonlinear Problem of Unsteady Heat Conduction of Layered Anisotropic Inhomogeneous Shells Under Boundary conditions of the First Kind on the Front Surfaces", Vestn. Samar. Gos. Tekhn. Univ., Ser. Fiz.-Mat. Nauki [J. Samara State Tech. Univ., Ser. Phys. \& Math. Sci.], 2014, no. 1(34), pp. 168-185. doi: 10.14498/vsgtu1281. (In Russian)

Author Details: Andrey P. Yankovskii (Dr. Phys. \& Math. Sci.), Leading Research Scientist, Lab. of Fast Processes Physics.

E-mail address: lab4nemir@rambler.ru 